SHORT REPORT

\title{
Motor evoked potentials from the pelvic floor in patients with multiple sclerosis
}

\author{
S Brostrom, J L Frederiksen, P Jennum, G Lose
}

J Neurol Neurosurg Psychiatry 2003;74:498-500

The use of motor evoked potentials (MEPs) to study the integrity of pelvic floor motor innervation is poorly described in the literature. This study evaluated the clinical use of pelvic floor MEPs in 16 women with multiple sclerosis. Lower urinary tract dysfunction was assessed with urodynamic investigations. Transcutaneous magnetic stimulation was applied over the motor cortex and spinal roots, and MEPs were recorded from the puborectalis, the external urethral sphincter, and the abductor hallucis muscles. In many patients, responses from the pelvic floor muscles could not be evoked, and central motor conduction times for the puborectalis motor pathways could only be calculated in $56 \%$. There was a poor correlation of abnormal conduction to lower urinary tract dysfunction. It is concluded, that unevokable responses from pelvic floor muscles in a patient with multiple sclerosis should be interpreted with care, and that pelvic floor MEPs have a limited clinical value in the investigation of suspected demyelinating disease.

$\mathrm{T}$ hough mostly used in the study of limb muscle innervation, transcranial magnetic stimulation (TMS) can

also be used to test the motor efferents of striated sphincters. However, most studies on the applicability of TMS in central motor conduction studies in patients with multiple sclerosis (MS) have targeted limb muscles. ${ }^{1-9}$ These studies have revealed one problem with the method: the fact that limb muscle responses to TMS are absent in a number of patients with MS. The sensitivity of TMS in patients with MS is another issue, as only two in three patients with clinically definite MS had abnormal central motor conduction times (CMCT). ${ }^{8}$ MEPs seem to have a limited diagnostic value in MS, and it is generally agreed that magnetic resonance imaging has superceded TMS in the diagnosis of MS. However, MEPs might still be more sensitive in revealing spinal cord lesions, and could thus have a role in detecting early phases of MS in patients with vague disturbances, for example, disturbances of sphincter function.'

It could also be argued that MEPs might be useful in investigating patients with idiopathic disturbances of sacral functions, for example, lower urinary tract dysfunction (LUTD), constipation, and sexual dysfunction. While pelvic floor dysfunction is prevalent among neurological patients, most female patients with LUTD have no overt neurological disease. In some cases of "idiopathic" LUTD, a subclinical neuropathy may be suspected, but not easily demonstrated. Assuming all cases of LUTD in patients with MS are neurogenic, a good correlation of pelvic floor CMCT to LUTD in these patients would support the clinical use of the method in investigations of cases of "idiopathic" LUTD, where a subtle neuropathy is suspected.

The aim of our study was twofold. Firstly, we wanted to assess the clinical value of pelvic floor MEPs by comparison with better defined lower extremity responses. Secondly, we wanted to correlate CMCT to LUTD, by studying MS patients with and without LUTD.

\section{METHODS}

Sixteen patients were recruited among the female patients of a large university MS clinic. All women had clinical definite MS according to the Poser criteria, ${ }^{10}$ with a mean disease duration of 6.6 years (range: 1.2-15.6). Patients with an EDSS $\geqslant 6.0$ were excluded. All patients had relapsing-remitting MS and received interferon beta treatment. The study population had a mean age of 43 years (range: $31-56$ ), a mean parity of 1.6 (range: 0-3), a mean height of $168 \mathrm{~cm}$ (range: 159-178), and a mean BMI of 24 (range: 18-35). Eight of the women were postmenopausal, one had had a hysterectomy, and seven received hormone replacement therapy. Patients taking drugs affecting neural conduction or neuromuscular transmission were excluded. None of the patients had clinical signs of peripheral neuropathies. The regional scientific ethical committee approved the study, and we obtained informed consent from all participants.

We used an adapted and validated version of the Bristol Female Lower Urinary Tract Symptoms questionnaire. ${ }^{11}$ Patients were scored as symptomatic if they had any lower urinary complaint more often than weekly. ${ }^{12}$ As urodynamic abnormalities has been described in patients with MS without lower urinary complaints, ${ }^{13}$ we performed urodynamics on all patients to reveal subclinical LUTD. Medium fill $37^{\circ} \mathrm{C}$ saline water cystometry with pressure-flow micturition studies were performed with patients in a sitting position. Kinesiological EMGs were recorded through hook wire electrodes in the external urethral sphincter (EUS). Urodynamic procedures and definitions conform to the standards recommended by the International Continence Society. ${ }^{14}$

MEPs were recorded with concentric needle electrodes from the EUS, right puborectalis muscle (PR), and with surface electrodes from the right abductor hallucis (AH). Amplification was $50 \mu \mathrm{V} /$ div for EUS, $100 \mu \mathrm{V} /$ div for PR, and $500 \mu \mathrm{V} / \mathrm{div}$ for $\mathrm{AH}$, bandpass was $20 \mathrm{~Hz}$ to $10 \mathrm{kHz}$ and the sweep-time 200 ms. Supramaximal magnetic stimuli were delivered as monophasic single pulses through a round $90 \mathrm{~mm}$ coil placed over the right hemisphere and over the right sacrum. For cortical stimulation we recorded MEPs in each of two conditions: with the target muscle relaxed and with moderate to maximum contraction (facilitation). MEPs were scored as abnormal if the CMCT was longer than reference values $+2 \mathrm{SD} .{ }^{15}$ Cortical latencies where used when CMCT could not be calculated. Normality was confirmed with the KolmogorovSmirnov test. A two tailed $t$ test was used to compare patients

Abbreviations: MEP, motor evoked potential; LUTD, lower urinary tract dysfunction; CMCT, central motor conduction time; MS, multiple sclerosis; TMS, transcranial magnetic stimulation; EUS, external urethral sphincter; $\mathrm{PR}$, puborectalis muscles; $\mathrm{AH}$, abductor hallucis muscle 


\begin{tabular}{|c|c|c|c|c|c|c|c|c|c|c|c|c|c|}
\hline \multicolumn{2}{|c|}{ Patients } & \multicolumn{3}{|c|}{$\begin{array}{l}\text { Neurological } \\
\text { impairment }\end{array}$} & \multicolumn{3}{|c|}{ Lower urinary tract symptoms } & \multicolumn{4}{|l|}{ Urodynamics } & \multicolumn{2}{|l|}{ MEPs } \\
\hline Age & $\begin{array}{l}\text { Disease } \\
\text { duration } \\
\text { (y) }\end{array}$ & EDSS & FS 1 & FS 5 & $\begin{array}{l}\text { urgency } \\
\text {-frequency }\end{array}$ & incontinence & $\begin{array}{l}\text { voiding } \\
\text { difficulty }\end{array}$ & $\begin{array}{l}\text { residual } \\
\text { urine }>100 \\
\mathrm{ml}\end{array}$ & $\begin{array}{l}\text { detrusor } \\
\text { hyperreflxia }\end{array}$ & DSD & $\begin{array}{l}\text { bladder } \\
\text { areflexia }\end{array}$ & $\begin{array}{l}\text { PR } \\
\text { abnormal }\end{array}$ & $\begin{array}{l}\mathrm{AH} \\
\text { abnormal }\end{array}$ \\
\hline 38 & 1.2 & 4.5 & 3 & 0 & - & - & + & + & - & + & - & - & - \\
\hline 40 & 4.6 & 4.0 & 3 & 0 & - & - & - & - & - & - & - & - & + \\
\hline 32 & 3.2 & 1.5 & 1 & 1 & - & - & - & - & - & - & - & + & + \\
\hline 38 & 4.2 & 3.5 & 2 & 2 & + & + & + & - & + & + & - & + & + \\
\hline 56 & 4.8 & 2.0 & 0 & 1 & + & + & - & - & - & - & - & - & - \\
\hline 53 & 1.3 & 5.5 & 3 & 2 & + & - & + & - & - & - & - & + & + \\
\hline 50 & 7.6 & 4.0 & 1 & 0 & - & - & - & - & - & - & - & + & + \\
\hline 43 & 13.1 & 5.0 & 3 & 2 & + & - & + & + & - & + & - & + & + \\
\hline 31 & 1.7 & 2.0 & 3 & 2 & + & + & - & - & + & - & - & - & - \\
\hline 49 & 7.5 & 4.0 & 2 & 4 & - & - & + & + & - & - & + & + & + \\
\hline 37 & 8.4 & 3.0 & 2 & 0 & - & - & - & - & - & - & - & + & + \\
\hline 53 & 11.9 & 2.5 & 2 & 1 & + & - & + & + & + & - & - & - & + \\
\hline 47 & 15.6 & 4.5 & 3 & 2 & + & + & + & + & + & + & - & N.A. & N.A. \\
\hline 42 & 6.4 & 5.0 & 3 & 0 & + & - & + & - & + & + & - & N.A. & N.A. \\
\hline 44 & 1.7 & 4.5 & 2 & 1 & + & - & + & - & - & - & - & - & - \\
\hline 41 & 11.7 & 2.5 & 1 & 2 & + & + & - & + & + & N.A & - & + & + \\
\hline
\end{tabular}

with healthy controls. A value of $\mathrm{p}<0.05$ was considered significant.

\section{RESULTS}

The mean CMCT of non-facilitated PR responses was $19.3 \mathrm{~ms}$ (95\% CI: 14.2 to 24.4 ); with facilitation CMCT was $18.4 \mathrm{~ms}$ (95\% CI: 13.3 to 23.5 ). For AH responses CMCT was $25.8 \mathrm{~ms}$ (95\% CI: 20.6 to 30.9 ) and $23.1 \mathrm{~ms}$ (95\% CI: 16.9 to 29.2 ) respectively. These values were all significantly prolonged compared with results from our studies of 30 healthy women. ${ }^{15}$ In 2 of 16 patients AH responses to cortical stimulation were absent despite facilitation. Even lower response rates to cortical stimulation were encountered with recordings from the pelvic floor muscles. Responses to spinal stimulation were also lacking in a number of patients, and consequently CMCT could only be calculated for PR in $56 \%$ and for AH in $75 \%$ of the patients. For EUS, CMCT could only be calculated in every fourth patient, and this variable was therefore excluded from further analyses.

Sixty three per cent of the patients complained of frequency-urgency, 56\% had voiding difficulties, and 31\% were incontinent (see table 1). Urodynamic studies were abnormal in $82 \%$ of symptomatic cases, and normal in all the asymptomatic cases. Sixty six per cent of the women with abnormal urodynamics also had an abnormal PR CMCT, and only half of the women with normal urodynamics had a normal PR CMCT.

\section{DISCUSSION}

The most striking finding in this study is the poor success we had with obtaining pelvic floor MEPs in MS patients. This experience concurs with findings in two previously published, smaller studies. Early studies at St Mark's Hospital in London used electrical scalp stimulation with recording of responses from the striated anal sphincter. ${ }^{316}$ They noted unrecordable responses in $38 \%$ of patients with MS and sacral dysfunction, probably reflecting the greater difficulty in recording low amplitude potentials from comparatively small striated muscles. Another British group used TMS to investigate a group of 10 patients with MS and LUTD, with recordings through needle electrodes in the EUS. ${ }^{17}$ They obtained responses in 5 of 10 patients, and in three of these five cases responses could only be detected with concomitant voluntary contraction of the sphincter. Unevokable lower limb responses to cortical stimulation in a proportion of MS patientscomparable to our finding for the $\mathrm{AH}$-have also been described. However, while non-responses in the lower limbs are rare in healthy controls, we found it in up to $83 \%$ of a group of 30 healthy women when examining pelvic floor muscles, ${ }^{18} \mathrm{a}$ finding we attribute to the small volume and deep inferomedial location of the cortical motor centre. The central organisation of pelvic floor motor control is complex and not completely understood, with pontine centres playing a central part besides the motor cortex. ${ }^{19}$ Intracerebral reorganisation attributable to MS plaques might affect these pathways, and further contribute to the difficulties in obtaining MEP responses. The success rate was increased with facilitation in our group of healthy controls, where we also verified the ability to contract the pelvic floor in all patients. ${ }^{15}$ (See fig 1). However, not all of our patients with MS could voluntarily contract the pelvic floor, a factor that might have affected the lack of increase in success rate with facilitation.

The low number of patients where CMCT could be calculated is also affected by a number of uninterpretable spinal MEPs. We have previously described the technical difficulties of sacral stimulation. ${ }^{18}$ We do not feel this problem is greater in MS patients, but it does compound analyses. In this study we attempted to remedy the problem by scoring MEPs as abnormal based on cortical latencies, where CMCT could not be calculated. Others have done likewise. ${ }^{7}$ However, this approach is questionable. Peripheral latencies-also for pelvic floor muscles-are dependent on the height of the patient ${ }^{15}$; this possible bias should at least be controlled. Furthermore, peripheral neuropathies should be excluded, which in practice would be difficult. CMCT is a relevant parameter when using MEPs in clinical decision making. Others have argued that a non-response in a patient with MS should be regarded as abnormal. ${ }^{48}$ B Based on our findings, we are not convinced this is a viable approach for the pelvic floor muscles, and we therefore believe the clinical value of the method is limited.

Our secondary aim was to correlate CMCT to LUTD. Assuming urodynamic abnormalities represent corticospinal lesions, the validity of PR CMCT was poor, with a nosographic sensitivity of $66 \%$ and a specificity of $50 \%$. Perhaps our initial assumption-that all cases of LUTD in MS patients are neurogenic-is wrong. LUTD is common in non-MS women of comparable age, ${ }^{12}$ and it would seem logical that also some female MS patients have LUTD on a non-neurogenic basis.

We conclude, that unevokable responses from pelvic floor muscles in patients with MS should be interpreted with care. Because unevokable responses are frequent, we believe that pelvic floor MEPs have a limited clinical value in the 
A

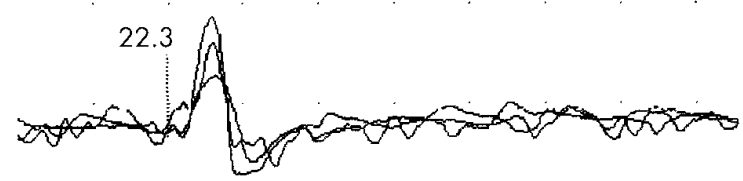

B

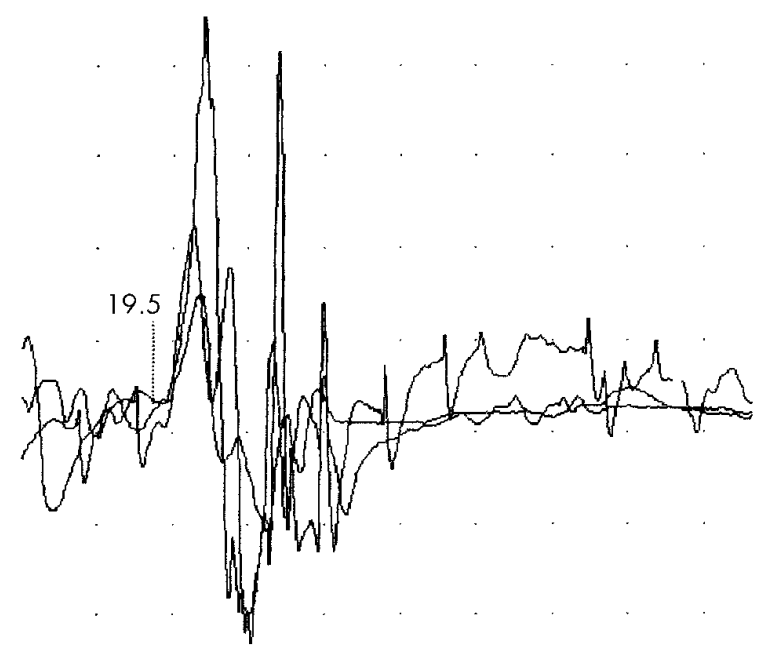

C

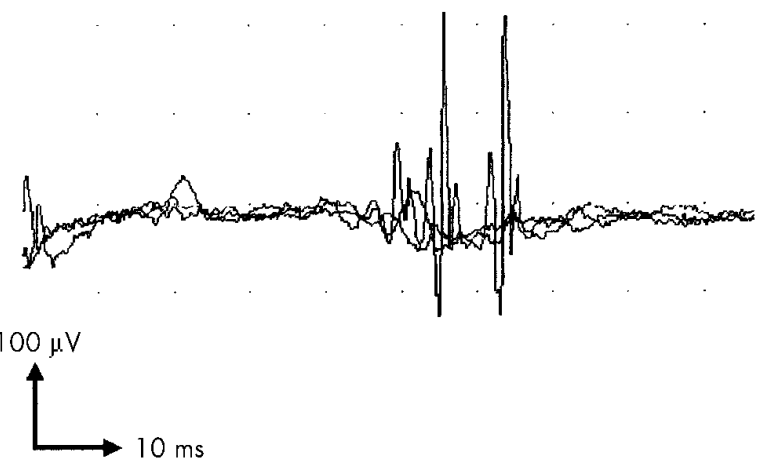

Figure 1 (A and B) Puborectalis MEPs in a 57 year old patient with a normal cystometry. Cortical stimulation with puborectalis relaxed and contracted (facilitation). (C) Unevokable responses from a 41 year old patient with detrusor hyperreflexia and a disease duration of 11.7 years. Note the capture of spontaneous motor unit potentials in the trigger windows, confirming correct placement of needle electrodes.

investigation of demyelinating disease, and a limited scientific value in the investigation of the pathophysiology of idiopathic LUTD.

\section{Authors' affiliations}

S Brostrom, G Lose, Department of Gynecology-Obstetrics, Glostrup Hospital, University of Copenhagen, Copenhagen, Denmark

J L Frederiksen, Department of Neurology, Glostrup Hospital, University of Copenhagen

P Jennum, Department of Clinical Neurophysiology, Glostrup Hospital, University of Copenhagen

Competing interests: none declared.

Correspondence to: Dr S Brostrom, Department of Gynecology-Obstetrics, Glostrup Hospital, DK-2600 Glostrup, Denmark; soren@brostrom.dk

Received 6 August 2002

Accepted in revised form 16 November 2002

\section{REFERENCES}

1 Hess CW, Mills KR, Murray NM, et al. Magnetic brain stimulation: central motor conduction studies in multiple sclerosis. Ann Neurol 1987;22:744-52.

2 Ingram DA, Thompson AV, Swash M. Central motor conduction in multiple sclerosis: evaluation of abnormalities revealed by transcutaneous magnetic stimulation of the brain. J Neurol Neurosurg Psychiatry 1988;51:487-94.

3 Mathers SE, Ingram DA, Swash M. Electrophysiology of motor pathways for sphincter control in multiple sclerosis. J Neurol Neurosurg Psychiatry 1990;53:955-60

4 Jones SM, Streletz L, Raab VE, et al. Lower extremity motor evoked potentials in multiple sclerosis. Arch Neurol 1991:48:944-8.

5 Kandler RH, Jarratt JA, Davies-Jones GA, et al. The role of magnetic stimulation as a quantifier of motor disability in patients with multiple sclerosis. J Neurol Sci 1991;106:31-4.

6 Mayr N, Baumgartner C, Zeithofer J, et al. The sensitivity of transcranial cortical magnetic stimulation in detecting pyramidal tract lesions in clinically definite multiple sclerosis. Neurology 1991;41:566-9.

7 Ravnborg $M$, Liguori $R$, Christiansen $P$, et al. The diagnostic reliability of magnetically evoked motor potentials in multiple sclerosis. Neurology 1992;42:1296-301.

8 Beer S, Rosler KM, Hess CW. Diagnostic value of paraclinical tests in multiple sclerosis: relative sensitivities and specificities for reclassification according to the Poser committee criteria. J Neurol Neurosurg Psychiatry 1995; 59:152-9.

9 Comi G, Leocani L, Medaglini S, et al. Evoked potentials in diagnosis and monitoring of multiple sclerosis. Electroencephalogr Clin Neurophysiol Suppl 1999;49:13-18.

10 Poser CM, Paty DW, Scheinberg L, et al. New diagnostic criteria for multiple sclerosis: guidelines for research protocols. Ann Neurol 1983;13:227-31.

11 Bernstein I, Sejr T, Abel l, et al. Assessment of lower urinary tract symptoms in women by a self-administered questionnaire: test-retest reliability. Int Urogynecol J Pelvic Floor Dysfunct 1996;7:37-47.

12 Moller LA, Lose G, Jorgensen T. The prevalence and bothersomeness of lower urinary tract symptoms in women $40-60$ years of age. Acta Obstet Gynecol Scand 2000;79:298-305.

13 Bemelmans BL, Hommes OR, Van Kerrebroeck PE, et al. Evidence for early lower urinary tract dysfunction in clinically silent multiple sclerosis. J Urol 1991;145:1219-24.

14 Abrams P, Blaivas JG, Stanton SL, et al. The standardisation of terminology of lower urinary tract function. The International Continence Society Committee on Standardisation of Terminology. Scand J Urol Nephrol Suppl 1988;114:5-19.

15 Brostrom S, Jennum P, Lose G. Motor evoked potentials from the striated urethral sphincter and puborectal muscle: normative values. Neurourol Urodyn (in press).

16 Snooks SJ, Swash M. Motor conduction velocity in the human spinal cord: slowed conduction in multiple sclerosis and radiation myelopathy. J Neurol Neurosurg Psychiatry 1985;48:1 135-9.

17 Eardley I, Nagendran K, Lecky B, et al. Neurophysiology of the striated urethral sphincter in multiple sclerosis. Br J Urol 1991;68:81-8.

18 Brostrom S, Jennum P, Lose G. Motor evoked potentials from the striated urethral sphincter: a comparison of concentric needle and surface electrodes. Neurourol Urodyn (in press).

19 Blok BF, Sturms LM, Holstege G. A PET study on cortical and subcortical control of pelvic floor musculature in women. J Comp Neurol 1997;389:535-44.

20 Kurtzke JF. Rating neurologic impairment in multiple sclerosis: an expanded disability status scale (EDSS). Neurology 1983;33:1444-52. 